\title{
Supplementary: i) Sepsis in Adult
}

\author{
Mitsuto Hasegawa, M D \\ Department of Medicine, School of Medicine, Keio University
}

At Keio University Hospital, septicemia was most frequently encountered in patients with hematological disorders such as leukemia and aplastic anemia, particularly in patientsswho developed serve granulocytopenia.

In aplastic anemia, there have been little variation in the occurrence of septicemia every year, while the incidence of septicemia has been increasing in acute leukemia since powerful anti-leukemic agents were introduced for treatment of acute leukemia. The majority of these septicemias occurred during induction therapy.

The main causative organisms were gram-negative rods, that is, Pseudomonas aeuruginosa, Klebsiella pneumoniae, E. coli and Enterobacter although the incidence of the causative organisms varies every year among gram-negative rods, depending upon the antibiotics available.

No case of septicemia was accompanied by disseminated intravascular coagulation in our series. Septic shock was one of the frequent complications in the past, but less frequently encountered in recent years.

Our regimens for treatment of septicemia were the combination of $\beta$-lactam and aminoglycoside anti-microbial agents. CZE and CBPG were the choice of $\beta$-lactam antibiotics, while GM or TOB were that of aminoglycoside antibiotics. All drugs were given intravenously and the dosage of CBPC (SBPC), GEZ, GM and TOB were 20-32 g, 8-12 g, 160-240 mg and 240-300 mg per day respectively. All patients were successfully treated with this regimen when the causative organism was susceptible to these antibiotics in vitro.

As far as the side effect of antibiotics was concerned, prolongation of bleeding time associated with inhibition of platelet functions (platelet aggregation and adhesiveness) was recorded in a patient with reticulum cell sarcoma when he was placed on CBPG, GEZ and GM as mentioned above. Discontinuation of the antibiotics resulted in improvement of platelet function. Platelet aggregation was also shown to be inhibited by CBPG in vitro.

\section{Supplementary: ii) Importance of Hemophilus Influenzae on Chronic Respiratory Infectious Diseases}

\author{
Keizo Matsumoto, M D \\ Department of Internal Medicine, Institute for Tropical Medicine, \\ Nagasaki University
}

We regard the pathogenic organisms detected more than $10^{7} / \mathrm{ml}$ by our quantitative culture method of sputum as the causative pathogens of infections. We summed up respiratory infectious diseases at the First Department of Internal Medicine of Tohoku University from 1971 to 1974 and those at the Department of Internal Medicine of the 